\title{
SULLA FLESSIONE INDOEUROPEA
}

Nella recensione, che ha dedicato ad un importante libro di E. Benveniste ${ }^{1}$, G. Bonfante osserva che "las formas véd. smáh imáh, etc., que son regulares, según su teoría, no las recuerda Benveniste" ${ }^{\prime 2}$. Dunque, forme del tipo scr. smáh., ie. *smés si possono analizzare perfettamente per mezzo della teoria di E. Benveniste che accoglie, sviluppandola ulteriormente, la proposta di J. Kuryłowicz ${ }^{3}$ : ie. ${ }^{*} s m e ́ s<*_{s-m-e ́ s}<*_{\text {zes- }}$ em-és. ${ }^{4}$

Se cosí è, ci troviamo di fronte ad un modello metodologico che, consentendo applicazioni non soltanto alla radice ma all'intera parola, potrebbe essere utilizzato per spiegare una buona parte della flessione, sia verbale sia nominale, indoeuropea. E gli esempii che seguono, sembrerebbero autorizzarlo.

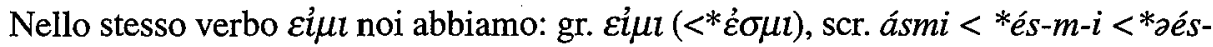
-em-eín; o con il verbo eĩ $\mu l$, scr. émi < éi-m-i<*aei-em-ei, e cosí le altre persone: gr. $\varepsilon \tilde{i} \zeta$

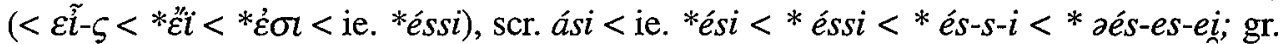

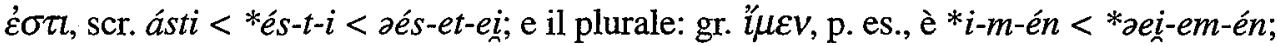
scr. imáh è *aei-em-és. E cosí di seguito. L'elemento tonico - radice o ampliamento conserva il grado forte, tutti gli altri si riducono a zero.

Un caso particolarmente interessante è quello di yunájmi $i^{5}$ : la flessione indoariana si adatta perfettamente allo schema di E. Benveniste, che elimina l'infisso nasale. Yunájmi ci dimostra che la radice non è né *ing - né *ieung-, ma senza dubbio *inu-, cioè

BENVENISTE 1935.

BONFANTE 1936, p. 163.

4 Se un po' di esagerazione c'è nell' affermazione che "tutto il 'laringalismo' non è in fondo che un tardo rigurgito della peggiore mentalità 'neogrammatica'" (BONFANTE 1957, p. 27), non c'è dubbio che la teoria 'laringale' comporti, in prospettiva storica, soluzioni spesso troppo dispendiose (oltre a BONFANTE 1936; 1937; 1944; 1945; 1957; cfr., tra i molti, almeno KRONASSER 1952; 1955) quando non in contraddizione con tutta la struttura fonematica dell'indoeuropeo (cfr. p. es., il $* g^{\mu} h$, che certo qualche problema di pronunciabilità lo dà, cui si oppone o ${ }^{\prime \prime} h^{z w}$ [MARTINET, p. 210] $0 * g^{z w} h$ [LEJEUNE, p. 38] o $* g^{z} w h$ [BENVENISTE 1937, p. 145]: tutti fomeni affricati!). Tuttavia, anche a noi sembra "economico per la teoria della radice indoeuropea considerare lo $a$ una 'semivocale' o se si vuole una 'semiconsonante' laringale" (ANCILLOTTI, p. 333). Di diversa opinione continua ad essere G. Bonfante (BONFANTE, 1970).

In parte interpretato dallo stesso E. Benveniste (BENVENISTE 1935, pp. 159-162). 
*ieu- e *-g- è il grado zero dell' ampliamento *ég- che appare sotto la sua forma piena in yunáj̆mi $<* i u-n-e ́ g-m-i<*_{i} e u-e n-e ́ g-e m-e i n$, non diversamente da yunákși $<* i u-n$ -ég-s-i< *ieu-en-ég-es-ei. Anche il pl. yunjmáh è regolare, perché risulta da *ieun-eneg-em-és che si riduce a *ịu-n-g-m-és; yunjánti deriva da *ieun-en-eg-én-et-ei $>*_{i} u-n$ -g-én-t-i. Le forme latine del singolare si spiegano nello stesso modo: iungō è *ieu-en-eg-ó̄ in cui $*_{-} \bar{o}$ - è il risultato della contrazione di $*_{-} e$ - con uno $*_{\partial-}$ - semiconsonantico ${ }^{6} \mathrm{e}$ corrisponde dunque a -ei-. -es-, -et-, ecc. Iungis, iungit sono ancora piú chiari: si tratta di *iu-n-g-és < *ieu-en-eg-és; *inu-n-g-ét-i < *ieun-en-eg-ét-eín, perchè iungit ha sicuramente perso la $-i$ finale ${ }^{7}$. Il pl. iungimus è piú difficile; deriva senz'altro da *iungomes, in cui tutto è chiaro, tranne l'ultima sillaba: *iungóm- < *ieu-en-eg-óm ${ }^{8}$. Iungitis è esattamente lo stesso caso: <*ieu-en-eg-ét-. Questo -es- finale potrebbe essere stato aggiunto per differenziare il plurale dal singolare.

Le forme in -óm- come lo *iungóm-, da noi proposto prima, sono regolari, sotto il profilo dell'alternanza, nell'aoristo forte: scr. bhujám $<* b h u-g-o ́ m<* b h e u$-eg-óm e cosí le altre persone: bhuǰás < *bheu-eg-és; bhuját < *bheu-eg-ét ${ }^{9}$.

Gli infiniti del tipo scr. drșé, bhuvé, ecc. si spiegano benissimo: si tratta di *der-ek-éi, *bheu-éi, ecc. e, probabilmente, hanno la stessa struttura anche gli infiniti 'passivi' e 'deponenti' latini del tipo $a g \bar{l}$, dì $c \bar{l}$. Dunque, sono proprio i verbi tematici che resistono maggiormente all'analisi; infatti un verbo come scr. bhárāmi, bhárasi è inspiegabile $^{10}$. Non bisogna, però, dimenticare che la coniugazione tematica è la piú recente.

La stessa situazione si coglie nei sostantivi. Infatti, se noi ci fermiamo sulla declinazione nominale piú antica, quella consonantica, avremo risultati sorprendenti. Prendiamo, p. es., il scr. rúč o il lat. dux. Il gen. ručás (lat. ducis) continua un ie. *luk $k^{u} e ́ s$, che deriva da *leun-ek $k^{u}-e ́ s$, perchè essendo l'ultimo elemento tonico, gli altri passano al

Secondo la cosí detta teoria di De Saussure-Benveniste. Infatti, "el carácter normativo, por decirlo así, de la teoría de Benveniste tiene como presupuesto la teoría de De Saussure sobre el carácter consonántico, o mejor semiconsonántico, de ə" (BONFANTE 1936, P. 160).

Cfr. BONFANTE 1935a.

Si incontrerà questa stessa desinenza al grado forte -os o -es (cfr. gr. dor. $\lambda \hat{\varepsilon} \gamma o \mu \varepsilon \varsigma$ ) che sembra aggiunta dopo le leggi dell'alternanza, nel plurale dei sostantivi: gr. $\pi \alpha \tau \tilde{\varepsilon} \rho \varepsilon \varsigma$, $\pi \circ \mu \mu \varepsilon v \varepsilon \varsigma$, scr. pitárah, ecc.

L'aumento, come si sa, interessa soltanto un numero ristretto di lingue indoeuropee dell'area meriodionale-orientale e cioè l'indoario (á-) l'armento $(e-)$ e il greco $(\dot{\varepsilon}-)$, che sono molto innovanti ed è un'aggiunta recente, tant'è che nei documenti piú antichi (Veda, Gāa $\theta \bar{a}$, poemi omerici) è facoltativo, come dimostra l'uso indifferente di ábharat e di bhárat nel sanscrito vedico o di ë $\varphi \varepsilon \rho \varepsilon$ e di $\varphi \varepsilon ́ \rho \varepsilon$ nel greco omerico. Un solo "caso pressoché certo" (NEGRI 1989b, p. 50) ha il miceneo: $a-p e-d o-k e$, da intendersi "quasi certamente" (NEGRI 1989a, p. 47) apedoke, è il gr. $\dot{\alpha} \pi \varepsilon \delta \omega \kappa \varepsilon$ Comunque, a poco a poco, l'uso dell'aumento per indicare il passato si fisserà in modo organico e diventerà obbligatorio nel sanscrito classico e nel greco postomerico (nell'armeno, invece, interesserà soltanto la 3 a persona singolare dell'aoristo; cfr. elik' 'lasciai').

Sono, almeno in parte, 'regolari' i verbi al grado zero (cfr. $\gamma \rho \alpha ́ \alpha \omega$ scr. tudáti, duháti, srjaati, avest. marazaiti, harazaiti, ecc.), ma essi sono relativamente rari e in parte tardi. 


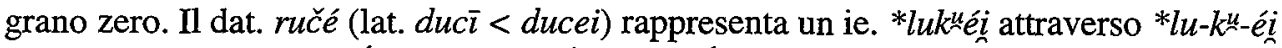

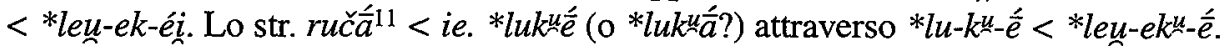

Anche i temi polisillabici sono in gran parte chiari.

*Pater- è una parola sicuramente antica. Il lat. patris (gen.) < ie. *potrés (gr. $\pi \alpha$ $\tau \rho o ́ \varsigma)$ è dunque < *pə-t-r-és <* pə-et-er-és, nella cui prima sillaba, l'elemento vocalico contenuto in $-\vec{a}$ - è caduto e l'elemento semiconsonantico $\partial$ si è vocalizzato in $\partial$ (scr. $\breve{l}$, lat. e gr. $\breve{a}$ ). Cosí il lat. patrī (dat.), scr. pitré sono un *pā-et-er-ée $>{ }^{*} p a-t-r$-éi.

Il scr. pitrá (strum.) è *pa-t-r-áa < *pa-et-er-áa. Il locativo sanscrito è pitári (gr.

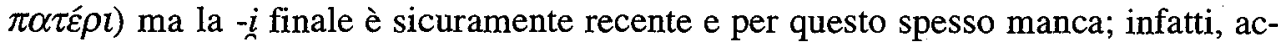
canto alle forme udáni, āsáni, akșáni, troviamo udán, āsán, akșán ${ }^{12}$. Una forma come pitár, udán, ecc. non offre alcuna difficoltà: c'è un ampliamento in meno e ciò deter-

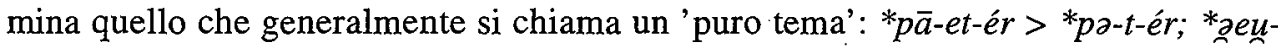
-ed-én > *u-d-én ${ }^{13}$.

Lo stesso metodo si può applicare, raggiungendo gli stessi risultati, ai temi in $-n^{-14}$ e a quelli in $-i$ - e in $-u^{-15}$.

Sono regolari anche i nominativi del tipo genu, pecu; scr. páśu, vásu, mádhu; gr.

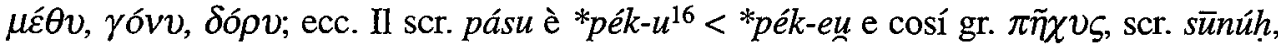
got. sunus. Nel tema, per ragioni evidenti, le alternanze si sono in generale perdute ${ }^{17}$ ma il genitivo conserva ancora bene l'alternanza -es/-s nella desinenza. Cosí, a parte l'alternanza del tema, il scr. sūnúh, got. sunus, lit. sūnùs, lat. manŭs da una parte e il scr. sūnóh, got. sunaus, lit. sūnaũs, lat. manūs dall' altra, rappresentano due tipi indoeuropei perfettamenre regolari: l'uno baritono (nom. *sŭ́nus), l'altro parossitono (gen. *sūnéus $<*^{*}$ su-en-éu-es) ${ }^{18}$.

11 La desinenza $-\bar{a}=$ ie. *-ée contiene un elemento vocalico $e$ e uno semiconsonantico 2.

12 Per il greco basta confrontare $\alpha \hat{i} \mathcal{F} \tilde{\varepsilon} v$, antico locativo di $\alpha \hat{i} \mathcal{F} \omega \dot{\omega}$.

13 Il caso è uguale a quello del vocativo greco $\pi \alpha ́ \alpha \varepsilon \rho$, il cui accento recente attesta che il nome è passato attraverso un periodo di atonia.

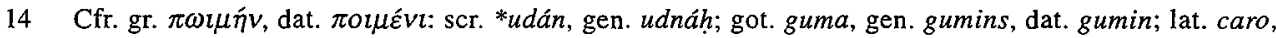
gen. carniss, dat. carnī $<{ }^{*}$ carnei.

15 Cfr. il dativo gr. $\pi \tilde{\eta} \chi \varepsilon \mathcal{F}$, scr. sūnávi; il genitivo got. kinnes < *genués, con la e del nom. genu,

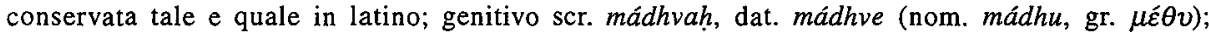
pasváh (nom. páśu); vasváh (nom. vásu, gr. om. ěv <*uésu), con lo spostamento dell'accento

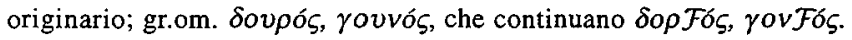

$16 \mathrm{E}$ cosí *pelu (radice *pel- del: lat. plēnus, gr. $\pi \dot{\imath} \mu \pi \lambda \eta \mu t$, scr. pūrnáh, ecc.) > got. filu. In germanico, sembra che ci siano anche dei nominativi del tipo *dr-éu-om, *gn-éu-om: got. tr-iu, ingl. tr-ee (cfr.

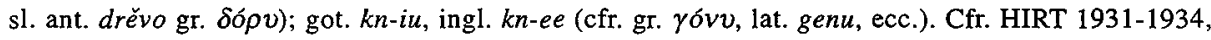
I, p. 57; II, pp. 38,42 .

17 Comunque abbiamo ancora dei genitivi di tipo arcaico come il scr. dróh (cfr. anche j̆nóh, snóh) < *dr-éu-s $<{ }^{*}$ der-éúu-es.

18 I participi presenti sono chiari, come dimostra, p. es., il sanscrito: gen. mřăatáh, dat. mrăaté, strum.

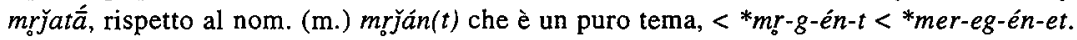


Ciò che abbiamo detto per i temi in $-u$, si applica perfettamente ai temi in $-i$ : lat. (nom.) ouis (< *óu-i-s, > *óu-ei-es), scr. ávih; lat. (gen.) ouis, scr. vés, con l'antica alternanza del tema.

Fra gli eterocliti, che rappresentano un tipo molto antico, si può citare, p. es., il lat. iter, dalla rad. *ei- 'andare', che deriva da un * ei-et-er. L'antico gen. *itnés (cfr., p. es., il scr. yákr-t, gen. yaknáh; ásr-k, asnáh) sarà l'esito di *ei-et-en-és; e cosí di seguito ${ }^{19}$. Il lat. iecur $(t)$ e il scr. yákrt rappresentano un *iék $k_{-r}^{u}-t<*_{i} e^{\prime} k_{-}^{u}-e r-e t$; il scr. ásrg e il gr.

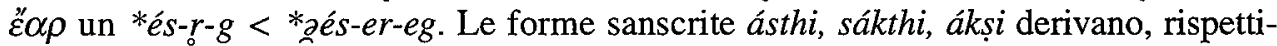
vamente, da *aés-eth-ei, * sék-eth-ei, * *ák $k_{-}^{u}-e s-e i^{20}$

Se già con queste ultime forme sanscrite ci siamo allontanti dalle posizioni di E. Benveniste ${ }^{21}$, dobbiamo ancora rilevare che, benché si registri qualche caso d'applicabilità: cfr. l'abl. pl. pitŕbhyyas < *po-t-r-bh-i-és < *pā-et-er-ebh-eí-és e l'abl. duale

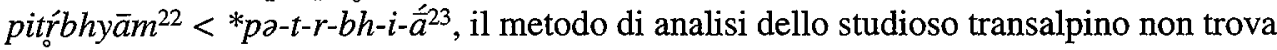
soddisfacente attuazione né nel plurale, né nel duale. E, crediamo, pour cause. $\mathrm{Il}$ plurale $^{24}$, infatti, (ma anche il duale) è una "creazione abbastanza recente" 25 , sicuramente posteriore al singolare e lo dimostrano bene, sia il fatto che soltanto in alcune lingue indoeuropee il duale ${ }^{26}$ è ben attestato e che, in altre, il plurale non ha la stessa omogeneità del singolare, ${ }^{27}$ sia precisi motivi fonologici ${ }^{28}$.

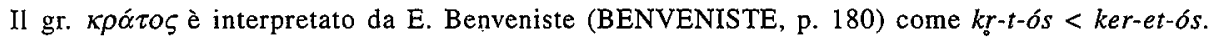
Egli, dunque, non ritiene fondata la proposta (Ibidem, n.1) di chi vuole vedere in $\kappa \rho \alpha \tau \varepsilon \rho o ́ \zeta, \kappa \rho \alpha \tau \delta \varsigma$ formazioni recenti (FRISK, s.u.).

Essi appartengono, come nominativi, alla stessa categoria del lat. genu, pecu, ecc. (cfr. supra, p. 3). BENVENISTE, p. 184.

La - $m$ è sicuramente un'aggiunta recente; cfr. lo sl. ant. pQť̌ma.

Queste forme polisillabiche (e cosí anche sūnúbhyah, sūnúbhyām, ecc.) erano ossitone ed hanno ritirato l'accento sulla penultima per una tendenza dell'indoario, scoperta da H.Hirt. E', cioè, quella che N.E. Collinge chiama la 'seconda legge di Hirt' (COLLINGE, p. 86). Cfr., comunque, HIRT 1895, p. 36 e HIRT 1902, p. 191 e, sopra tutto, HIRT 1904.

24 E' probabile che, nella sua fase piú antica, l'indoeuropeo esprimesse l'idea di plurale' attraverso mezzi sintattici e non morfologici, ricorrendo cioè, come proponeva H. Hirt (HIRT 1921-1937, VI, pp. 21 segg.), al singolare collettivo, ad avverbi, ad aggettivi e numerali aggiunti al sostantivo, alla ripetizione, ecc. ma, sopra tutto, al suppletivismo (tipo: russo čelověk: ljudi). E che questo sia un tratto arcaico lo dimostra anche il fatto che un tale procedimento domina in tutte le lingue indoeuropee nella formazione del plurale dei pronomi personali, che rappresentano notoriamente uno degli elementi piú antichi.

Abbiamo messo tra apici l'espressione 'idea di plurale', perchè in realtà è inesatta. Non avendo, infatti, la forma del plurale, l'indoeuropeo piú antico non doveva neppure possedere la nozione e, doveva categorizzare in modo diverso quella realtà fenomenica che noi esprimiamo per mezzo del plurale. Il che vuol dire, dal punto di vista del linguista, che questo complesso d'idee era interpretato in modo diverso dal nostro.

MARTINET, p. 202.

E' presente soltanto nel sistema fonologico dell'indoario, del greco antico - specialmente del dialetto attico - dello slavo antico e del lituano, mentre in quello delle altre lingue non si trovano che rare tracce. Nelle lingue germaniche, p. es., il gotico conserva tracce del duale nel verbo (prima 
Sempre per lo stesso motivo, cioè perché piú recenti, le cose non vanno meglio nelle declinazioni dei temi in $-o^{-29}$ e di quelli in $-\bar{a}^{-30}$.

Invece i participi e gli aggettivi non creano problemi: i participi presenti sono chiari $^{31}$ e cosí quelli in -tó-s, originariamente ossitoni ${ }^{32}$; e anche gli aggettivi, che sembrano avere avuto la stessa accentazione ${ }^{33}$ in indoeuropeo ${ }^{34}$, si possono analizzare con facilità; il gr. $\lambda v \gamma \rho o ́ \zeta$, p. es., deriva da $* l u$-g-r-ós < *leun-ed-er-ós $s^{35}$.

Queste poche osservazioni confermano, crediamo, ciò che $\mathrm{G}$. Bonfante ${ }^{36}$ aveva già sostenuto in alcuni suoi lavori ${ }^{37}$, cioè che la flessione indoeuropea è composta da elementi molto disparati e che non è quasi mai possibile stabilire un limite netto, dal punto di vista storico, tra ampliamento, suffisso, tema, desinenza. Dunque, la teoria, che E. Benveniste ha formulato per le radici, le basi e gli ampliamenti, trova perfetta applica-

e seconda persona della forma attiva), nel pronome di prima e, relativamente ai casi obliqui, di seconda persona, nell'aggettivo numerale ahtau. Nell'inglese antico, nel sassone antico e nel nordico antico, l'uso del duale è limitato quasi esclusivamente ai pronomi di prima e seconda persona, mentre il "il ne reste plus de trace en vha. à l'exception de unker 'nous deux,' attesté une seule fois chez Otfried 3.22.32" (JOLIVET-MOSSE, p. 104).

27 P.es., "a juzgar por el hetita, [la oposición de número sg./pl.] es antigua en N. y Ac., menos en G. y reciente en los demás casos; pero incluso en $N$. y Ac. hay datos a favor de su carácter secundario: N.sg. por N. pl. en hetita, derivación del N. y Ac. pl. a partir de los de singular" (ADRADOS, p. 507).

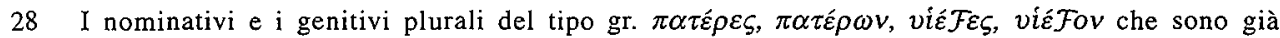
indoeuropei (cfr. scr. pitấrah, pitārām; lat. hostês, hostium; got. gasteis, gaste, sl. ant. synove, synovŭ) non entrano nel sistema di E. Benveniste (BENVENISTE 1935), né in quello di $\mathrm{H}$. Hirt (HIRT 1921-1937, V), né in nessun altro sistema di apofonia indoeuropea. Sembra chiaro, dunque, che *-ěs, *ôm sono delle aggiunte recenti; infatti, se li si elimina, tutto funziona alla perfezione; gr.

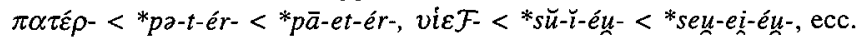

29 Cfr. STREITBERG, p. 310; HIRT 1931-1934, II, p. 28 (ma anche pp. 38, 49). Tutt'al piú si potrebbe dividere *ingóm (lat. iugum, ecc.) in *ieun-eg-óm > *ịu-g-óm, ma per gli altri casi le difficoltà sono insormontabili.

30 Come ha visto J. Schmidt (SCHMIDT, pp. 117 e segg.), soltanto il nominativo con valore collettivo è antico. E' ben vero che un paradigma del tipo nom. $\pi \tau v \chi \bar{\eta}$, gen. $\pi \tau v \chi o ́ \varsigma$ dat. $\pi \tau v \chi i ́$ o nom. $\varphi v \gamma \dot{\eta}$, acc. $\varphi \hat{v} \gamma \alpha \delta \varepsilon$ s'incontra ancora in Omero, ma è sicuramente un tratto arcaico (cfr. BONFANTE 1935 c, in particolare, p. 260 e n.1). Ora, un nom. $\varphi v \gamma \eta \dot{~ s ' i n t e r p r e t a ~ c o n ~ l a ~ t e o r i a ~ d i ~ E . ~ B e n v e n i s t e: ~}$ $\varphi v \gamma \dot{\eta}<* b h u-g-\dot{a}<* b h e \underline{a}-e g-\bar{a}$ (sull'⿳亠丷ă, cfr. anche supra n.11).

31 P. es., cfr. il scr. mry̌atáh (gen.), mry̌até (dat.), mry̌atá (strum.). Il nom. m. mry̆ăn(t) è un puro tema: *mer-eg-én-et $>*_{m r}$-g-én-t.

32 P.es., cfr. il lat. dĭctus $<* d \tilde{t}$-k-t-ós $<*$ dei-ek-et-ós.

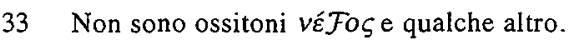

34 Cfr. HIRT 1921-1937, V, pp. 268-280.

35 L'elemento -er- si trova, p. es., nel lat. iter $\left(<*_{i-t-e ́ r}<* e i\right.$-et-ér $)$, dove alterna con -en-nel gen. it-in-er-is.

36 Ma già H. Hirt (HIRT 1921-1937, III, p. 267) forse l'aveva intravisto, quando chiama "Basis" una parola come il scr. tulá, la cui $\bar{a}$, dunque, non è tematica!

P.es., cfr. BONFANTE 1935b, 1935c. 
zione, in una gran qualità di casi, e precisamente nei paradigmi piú antichi, anche a ciò che chiamiamo desinenza. ${ }^{38}$

\section{BIBLIOGRAFIA CITATA}

ANCILLOTTI: A. Ancillotti, Elogio del variabile. Introduzione alla linguistica storica: l'indoeuropeistica, Milano, 1988.

ADRADOS: F.R. Adrados, Lingüística indoeuropea, Madrid, 1975.

BENVENISTE 1935: E. Benveniste, Origines de la formation des noms en indoeuropéen. I., Paris, 1935.

BENVENISTE 1937: E. Benveniste, Le problème $d u * \mathrm{o}$ indoeuropéen "Bulletin de la Société de Linguistique de Paris", 381 (1937), pp. 139-147.

BONFANTE 1935a: G. Bonfante, -i final en latin, "Zeitschrift für vergleichende Sprachforschung auf dem Gebiete der indogermanischen Sprache", 62 (1935), pp. 265-267.

BONFANTE 1935b: G. Bonfante, El origen del nominativo singular sigmático indoeuropeo, "Emerita", 3/2-1 (1935), pp. 56-76.

BONFANTE 1935c: G. Bonfante, Sobre de la función de la heteróclisis en la formación de los temas nominales indoeuropeos, "Emerita", 3/2(1935), pp. 257-276.

BONFANTE 1936: G. Bonfante, rec. a E. Benveniste, Origines de la formation des nom en indoeuropéen. I., Paris, 1935, "Emerita", 4/1 (1936), pp. 157-164.

BONFANTE 1937: G. Bonfante, rec. a J. Kuryłowicz, Etudes indoeuropéennes. I., Cracovia, 1934, "Emerita", 5/2 (1937), pp. 165-167.

BONFANTE 1944: G. Bonfante, rec. a E.H. Sturtevant, The Indo-Hittite laryngeals, Baltimora, 1942, "Classical Philology", 39 (1937) pp. 51-57.

BONFANTE 1945: G. Bonfante, A retort, "Classical Philology", 40 (1945), pp. 161165.

BONFANTE 1957: G. Bonfante, La teoria laringale, "Paideia", 12/1 (1957), pp. 22-28.

BONFANTE 1970: G. Bonfante, Il tipo delle radici indoeuropee, in Mille. I dibattiti del Circolo Linguistico Fiorentino 1945-1970, Firenze, 1970, pp. 19-26.

COLLINGE: N.E. Collinge, The law of Indo-European, Amsterdam-Philadelphia, 1985.

HIRT 1895: H. Hirt, Der indogermanische Akzent: Ein Handbuch, Strassburg, 1895.

HIRT 1902: H. Hirt, Handbuch der griechischen Laut- und Formenlehre, Heidelberg, 1902.

HIRT 1904: H. Hirt, Zur Entstehung der griechischen Betonung, "Indogermanische Forschungen", 16 (1904), pp. 71-92.

38 Cfr. ciò che scrive J. Kuryłowicz: "Rien ne nous autorise à croire a priori que les désinences de déclinaison se comportent autrement que les suffixes ordinaires. Et en effet un examen des langues historiques prouve que les règles concernant la forme des complexes et des suffixes s'appliquent aussi aux cas nominaux et aux désinences". (KURY€OWICZ 1935, p. 135). 
HIRT 1931-1934: H. Hirt, Handbuch der Urgermanischen, Heidelberg, 1931-1934, voll. I-III.

HIRT 1921-1937: H. Hirt, Indogermanische Grammatik, Heidelberg, 1921-1937, voll. I-VII.

JOLIVET-MOSSE: A. Jolivet-F. Mossé, Manuel de l'allemand du Moyen Age des origines au XIV sièce, Paris, 1972.

KRONASSER 1952: H. Kronasser, 'Structural linguistic' und Laryngal-Theorie, in Studien zur indogermanischen Grundsprache, herausgegeben von W. Brandenstein, Graz, 1925, pp. 56-71.

KRONASSER 1955: H. Kronasser, Vergleichende Laut- und Formenlehre des Hethitischen, Heidelberg, 1955.

KURYŁOWICZ 1927: J. Kuryłowicz, $\exists$ indoeuropéen et h hittitte, in Symbolae Io. Rozwadowski, Cracovia, 1927, I, pp. 95-104.

KURYŁOWICZ 1935: J. Kuryłowicz, Etudes indo-européennes, Cracovia, 1935.

LEJEUNE: M. Lejeune, Phonétique historique du mycénien et du grec ancien, Paris, 1987.

MARTINET 1968: A. Martinet, Economia dei mutamenti fonetici. Trattato di fonologia diacronica. Introduzione e traduzione di G. Caravaggi, Torino, 1968 [trad. di: Bern, 1955].

MARTINET 1987: A. Martinet, L'indoeuropeo. Lingue, popoli e culture, Bari, 1987. [trad. di: Paris, 1986].

NEGRI 1989a: M. Negri, Le cronologie di Cnosso: argomenti linguistici "Atti del Sodalizio Glottologico Milanese", XXX (1989) (ma 1992), pp. 43-53.

NEGRI 1989b: M. Negri, Linee d'innovazione e aree di conservazione del greco di età micenea, in Innovazione e conservazione nelle lingue. Atti del Convegno della Società Italiana di Glottologia, Messina, 9-11 novembre 1989. Testi raccolti a cura di V. Orioles, Pisa, 1991, pp. 35-75.

SCHMIDT: J. Schmidt, Die Pluralbildungen der indogermanischen Neutra, Weimar, 1989.

STREITBERG: W. Streitberg, Urgermanische Grammatik. Einführung in des vergleichende Studium der altgermanischen Dialekte, Heidelberg, 1974 [quarta ristampa inalterata di: 1896$]$.

Povzetek

O PREGIBANJU V INDOEVROPŠČINI

Opombe k recenziji G. Bonfanteja Benvenistovega dela Origines de la formation des noms en indoeuropéen skušajo potrditi, da more Benvenistova teorija o korenih razložiti tudi dobršen del pregibanja v indoevropščini, tako za glagol kot za samostalnik. 